\title{
Scaling relations in large-Prandtl-number natural thermal convection
}

\author{
Olga Shishkina* \\ Max Planck Institute for Dynamics and Self-Organization, Am Fassberg 17, 37077 Göttingen, Germany \\ Mohammad S. Emran \\ Max Planck Institute for Dynamics and Self-Organization, Am Fassberg 17, 37077 Göttingen, Germany
}

Siegfried Grossmann

Fachbereich Physik der Philipps-Universität, Renthof 6, 35032 Marburg, Germany

\begin{abstract}
Detlef Lohse
Physics of Fluids group, Department of Science and Engineering, Mesa+ Institute, Max Planck Center for Complex Fluid Dynamics and J. M. Burgers Centre for Fluid Dynamics, University of Twente, P.O. Box 217, 7500 AE Enschede, The Netherlands

and Max Planck Institute for Dynamics and Self-Organization, Am Fassberg 17, 37077 Göttingen, Germany
\end{abstract}

(Received 17 May 2017; published 25 October 2017)

\begin{abstract}
In this study, we follow Grossmann and Lohse [Phys. Rev. Lett. 86, 3316 (2001)], who derived various scalings regimes for the dependence of the Nusselt number $\mathrm{Nu}$ and the Reynolds number Re on the Rayleigh number Ra and the Prandtl number Pr. We focus on theoretical arguments as well as on numerical simulations for the case of large-Pr natural thermal convection. Based on an analysis of self-similarity of the boundary layer equations, we derive that in this case the limiting large-Pr boundary-layer dominated regime is $\mathrm{I}_{\infty}^{<}$, introduced and defined by Grossmann and Lohse [Phys. Rev. Lett. 86, 3316 (2001)], with the scaling relations $\mathrm{Nu} \sim \operatorname{Pr}^{0} \mathrm{Ra}^{1 / 3}$ and $\operatorname{Re} \sim \operatorname{Pr}^{-1} \mathrm{Ra}^{2 / 3}$. Our direct numerical simulations for Ra from $10^{4}$ to $10^{9}$ and $\operatorname{Pr}$ from 0.1 to 200 show that the regime $\mathrm{I}_{\infty}^{<}$is almost indistinguishable from the regime $\mathrm{III}_{\infty}$, where the kinetic dissipation is bulk-dominated. With increasing Ra, the scaling relations undergo a transition to those in $\mathrm{IV}_{u}$ of Grossmann and Lohse [Phys. Rev. Lett. 86, 3316 (2001)], where the thermal dissipation is determined by its bulk contribution.
\end{abstract}

DOI: 10.1103/PhysRevFluids.2.103502

\section{INTRODUCTION}

Thermal convection flows are common in nature and technology. One of the classical systems to study such flows is Rayleigh-Bénard convection (RBC) [1-4], where a fluid is confined between a heated bottom plate and a cooled top plate. In RBC the main input or control parameters of the system are the Rayleigh number $\operatorname{Ra} \equiv \alpha g \Delta H^{3} /(\kappa \nu)$, the Prandtl number $\operatorname{Pr} \equiv \nu / \kappa$, and the cell geometry. Here $v$ denotes the kinematic viscosity, $\kappa$ the thermal diffusivity, $\alpha$ the isobaric thermal expansion coefficient of the fluid, $g$ the acceleration due to gravity, $H$ the distance between the top and bottom plates, and $\Delta \equiv T_{+}-T_{-}>0$ with $T_{+}$and $T_{-}$the temperatures of, respectively, the heated bottom and the cooled top plates.

The main global response characteristics of this convective system are the mean convective heat transport from bottom to top and the mean momentum transport by convection, which are represented, respectively, by the Nusselt number $\mathrm{Nu}$ and the Reynolds number Re. How $\mathrm{Nu}$ and $\mathrm{Re}$ depend on $\mathrm{Ra}$ and $\mathrm{Pr}$ is the main issue in investigations of thermally driven flows.

The kinetic and the thermal dissipation rates are fundamental concepts in turbulent thermal convection. For some convective flow configurations, including RBC, it is possible to derive

\footnotetext{
*olga.shishkina@ds.mpg.de
} 
analytical relations for the time- and volume-averaged kinetic and thermal dissipation rates $\epsilon_{u}$ and $\epsilon_{\theta}$ in terms of Ra and Nu cf. [1,5]. For example, in RBC, it holds:

$$
\begin{gathered}
\epsilon_{u}=\left(v^{3} / H^{4}\right)(\mathrm{Nu}-1){\operatorname{Ra~} \operatorname{Pr}^{-2},}, \\
\epsilon_{\theta}=\left(\kappa \Delta^{2} / H^{2}\right) \mathrm{Nu} .
\end{gathered}
$$

Using these relations, Grossmann and Lohse developed a scaling theory (GL theory) [5-11], which is based on a decomposition of $\epsilon_{u}$ and $\epsilon_{\theta}$ into their boundary-layer (BL) and bulk contributions. Basically, the so-called scaling regimes I, II, III, and IV in the GL theory are associated with the BL-BL, bulk-BL, BL-bulk, and bulk-bulk dominance in $\epsilon_{u}$ and $\epsilon_{\theta}$, respectively. The assigned subscripts $u$ and $\ell$ to these regimes indicate the $u$ pper-Pr and $\ell$ ower-Pr cases, respectively. Equating $\epsilon_{u}$ and $\epsilon_{\theta}$ to their estimated either bulk or BL contributions and employing the Prandtl-Blasius BL theory [12-15] for the thermal and viscous BL thicknesses, theoretically possible limiting scaling regimes followed. In particular, for the case of large Pr-number thermal convection, the various possible scaling regimes $\mathrm{I}_{u}, \mathrm{I}_{\infty}^{<}, \mathrm{I}_{\infty}^{>}, \mathrm{II}_{u}, \mathrm{III}_{u}, \mathrm{III}_{\infty}, \mathrm{IV}_{u}$ were obtained, see Grossmann and Lohse [6] for the details.

In this paper, we first derive a scaling relation between $\mathrm{Re}, \mathrm{Nu}$, and $\mathrm{Pr}$ in natural boundarylayer dominated thermal convection, in which the complete convective flow is due to temperature differences without any externally controlled wind input. This relation implies that in RBC the limiting large-Pr boundary-layer dominated regime is $\mathrm{I}_{\infty}^{<}$, with the scaling relations $\mathrm{Nu} \sim \operatorname{Pr}^{0} \mathrm{Ra}^{1 / 3}$ and $\mathrm{Re} \sim \mathrm{Pr}^{-1} \mathrm{Ra}^{2 / 3}$. This regime $\mathrm{I}_{\infty}^{<}$matches the regime $\mathrm{III}_{\infty}$ for larger $\mathrm{Ra}$, which in turn adjoins the regime $\mathrm{IV}_{u}$ for even higher Rayleigh numbers. Based on the results of our direct numerical simulations (DNS) of RBC in a cylindrical container of aspect ratio 1 , for Ra ranging from $10^{4}$ to $10^{9}$ and Pr from 0.1 to 200 , we demonstrate the correctness of the derived scaling relations in regime $\mathrm{I}_{\infty}^{<}$and also the transition to regime $\mathrm{IV}_{u}$ for sufficiently large Ra.

\section{RELATION BETWEEN Re, Nu, AND Pr IN NATURAL (PURELY THERMALLY DRIVEN) BOUNDARY-LAYER DOMINATED THERMAL CONVECTION}

Following Prandtl [12], we consider a fluid flow along a heated plate and choose the coordinate system such that the $x$ direction is along the plate and the $z$ direction is pointing vertically away from the plate. We assume that the mean flow in the other horizontal direction $(y)$ is much weaker than that in $x$ or $z$ and, therefore, consider a two-dimensional flow that depends on $x$ and $z$ only. Under the standard Prandtl BL approximation [14,15] we obtain the following momentum Eq. (3) and thermal Eq. (4) BL equations for a fluid motion near the isothermally heated horizontal plate:

$$
\begin{gathered}
u_{x} \partial_{x} u_{x}+u_{z} \partial_{z} u_{x}=v \partial_{z}^{2} u_{x}+\beta \alpha g\left(T-T_{0}\right), \\
u_{x} \partial_{x} T+u_{z} \partial_{z} T=\kappa \partial_{z}^{2} T,
\end{gathered}
$$

where $T(x, z)$ denotes the temperature field above the plate, $\left(u_{x}, u_{z}\right)$ is the velocity vector in the coordinates $(x, z)$ and in the buoyancy term $\beta \alpha g\left(T-T_{0}\right)$ we have $\beta$ vanishing in the case of a horizontal heated plate and $\beta=1$ in the case of vertical heated plate. The temperature boundary conditions are

$$
T(x, 0)=T_{+}, \quad T(x, \infty)=T_{0} \equiv\left(T_{+}+T_{-}\right) / 2,
$$

and the velocity vanishes at the plate,

$$
u_{x}(x, 0)=u_{z}(x, 0)=0
$$

due to the assumed no-slip boundary conditions. In the classical Prandtl-Blasius approach [12-14], a constant flow above an infinite horizontal plate is considered, which is parallel to the plate. This leads to the next boundary condition for the velocity, far away from the plate, $u_{x}(x, \infty)=U \neq 0$, 
where $U$ is the so-called wind outside the BL. The resulting Prandtl-Blasius approximation of the thermal boundary layer in laminar thermal convection is widely used, see, e.g., Refs. [5,16]. This approach can be generalized to include the influence of the turbulent fluctuations within the BL [17-19] and also can be adapted to the case of a nonvanishing pressure gradient within the BL (or to the case of a non-constant wind above the horizontal isothermal plate, which approaches the plate at arbitrary angle), see Refs. [20,21]. In all these cases the wind does not vanish in the bulk and, generally speaking, is artificially imposed in the boundary layer equations.

In natural thermal convection, where no mean flow is imposed in the core part of the domain, all the wind above the BL is self-organized. At the plate its absolute value is equal to zero. With growing distance from the plate, the absolute value of the wind first increases and, after it has achieved its maximum, slowly vanishes back to zero. The maximum value of the wind can be then considered as the reference quantity $U$. The magnitude of $U$ and the location, where the maximum of the wind is achieved, depends implicitly on Ra and Pr. Such approach was considered for the case of vertical convection (between two isothermal differently heated vertical walls) in Shishkina [22]. Here we use the ideas from Ref. [22] to derive a relation between the Nusselt number $\mathrm{Nu}$ and the Reynolds number Re in laminar natural convection for the case, where gravity is orthogonal to the isothermally heated plate, i.e., to the RBC case.

The idea of the method suggested in Ref. [22] is the following. Let us construct a similarity variable $\xi$ as the usual combination of the vertical and horizontal coordinates, but now also with introduced Prandtl number and Rayleigh number:

$$
\xi=\operatorname{Pr}^{a} \operatorname{Ra}^{b}(x / L)^{c}(z / H) .
$$

Here $L$ is the length of the heated plate and the exponents $a, b$, and $c$ are not fixed for the time being. In a similar way we introduce the exponents $d, e$, and $f$ for, respectively, $\operatorname{Pr}, \operatorname{Ra}$, and $x$ into the stream-function $\Psi$ :

$$
\Psi=\nu \operatorname{Pr}^{d} \operatorname{Ra}^{e}(x / L)^{f} \phi(\xi),
$$

from which the velocity components in horizontal and vertical directions can be evaluated as $u_{x}=\partial_{z} \Psi$ and $u_{z}=-\partial_{x} \Psi$. The temperature $T$ is represented though a nondimensional temperature $\theta$ as follows:

$$
T=T_{0}+\left(T_{+}-T_{0}\right) \theta(\xi)=T_{0}+(\Delta / 2) \theta(\xi) .
$$

The Nusselt number can then be calculated as

$$
\mathrm{Nu} \equiv \frac{-\left.L^{-1} \int_{0}^{L} \kappa \partial_{z} T\right|_{z=0} d x}{\kappa \Delta / H}=\frac{-\theta^{\prime}(0)}{2(c+1)} \operatorname{Pr}^{a} \operatorname{Ra}^{b},
$$

where $\theta^{\prime} \equiv d \theta / d \xi$ is the derivative of $\theta$ with respect to the similarity variable $\xi$. Analogously, we can derive the Reynolds number, which is defined in terms of the maximal mean velocity along the heated plate:

$$
\operatorname{Re} \equiv U H / \nu, \quad U \equiv \max _{z} L^{-1} \int_{0}^{L} u_{x} d x .
$$

Here the horizontal velocity $u_{x}$ equals

$$
u_{x}=\partial_{z} \Psi=\operatorname{Pr}^{a+d} \operatorname{Ra}^{b+e}(v / H)(x / L)^{c+f} \phi^{\prime} .
$$

Assuming that the maximal velocity is achieved at a certain value of $\xi=\hat{\xi}$, where $\phi^{\prime \prime}(\hat{\xi})=0$, from Eqs. (11) and (12) we obtain

$$
\operatorname{Re}=\operatorname{Pr}^{a+d} \operatorname{Ra}^{b+e}(c+f+1)^{-1} \phi^{\prime}(\hat{\xi}) .
$$


This together with Eq. (10) yields

$$
\frac{\mathrm{Nu}}{\operatorname{Re}^{1 / 2} \operatorname{Pr}^{1 / 2}}=-\frac{\sqrt{c+f+1}}{2(c+1)} \frac{\theta^{\prime}(0)}{\left[\phi^{\prime}(\hat{\xi})\right]^{1 / 2}} \operatorname{Pr}^{(a-d-1) / 2} \operatorname{Ra}^{(b-e) / 2} .
$$

Now we substitute Eqs. (7)-(9) into the BL energy Eq. (4) and require the independence of the resulting BL equation from $\operatorname{Pr}$ and $\mathrm{Ra}$. The equation then takes the following form:

$$
\operatorname{Pr}^{a} \operatorname{Ra}^{b}(x / L)^{c}(1 / H) \theta^{\prime \prime}+f \operatorname{Pr}^{d+1} \operatorname{Ra}^{e}(x / L)^{f-1}(1 / L) \phi \theta^{\prime}=0,
$$

which implies

$$
d=a-1, \quad e=b, \quad f=c+1 .
$$

The BL energy Eq. (15) then reduces to

$$
\theta^{\prime \prime}+\frac{c+1}{\Gamma} \phi \theta^{\prime}=0
$$

with the aspect ratio $\Gamma \equiv L / H$. Note that the boundary conditions for $\phi$ and $\theta$ are also independent of $\operatorname{Pr}$ and Ra, i.e., $\phi(0)=\phi^{\prime}(0)=0, \phi^{\prime}(\infty)=0$, and $\theta(0)=1, \theta(\infty)=0$, which one can obtain from Eqs. (5)-(9).

With Eq. (16), the relation (14) is reduced to

$$
\frac{\mathrm{Nu}}{\operatorname{Re}^{1 / 2} \operatorname{Pr}^{1 / 2}}=-\frac{1}{\sqrt{2(c+1)}} \frac{\theta^{\prime}(0)}{\left[\phi^{\prime}(\hat{\xi})\right]^{1 / 2}} \operatorname{Pr}^{0} \operatorname{Ra}^{0} .
$$

The momentum Eq. (3) with the similarity variable Eq. (7) and the stream-function Eq. (8) with the constants Eq. (16) takes the form

$$
\operatorname{Pr}^{4 a} \phi_{\xi \xi \xi}+\operatorname{Pr}^{4 a-1}\left[(c+1) \phi \phi_{\xi \xi}-(2 c+1)\left(\phi_{\xi}\right)^{2}\right]-\beta \operatorname{Ra}^{1-4 b}(x / H)^{-4 c-1} \theta / 2=0 .
$$

With a proper choice of the constant $b(b=1 / 4$ for $\beta \neq 0)$ this equation can be released from the Ra-dependence. However, as one can see from the comparison of the first two terms in Eq. (19), it cannot be made generally independent of Pr. However, for $\operatorname{Pr} \gg 1$, the second term in Eq. (19) becomes negligible compared to the first term, which means that for $\operatorname{Pr} \rightarrow \infty$ there exist certain values of $a, b, c, d, e$, and $f$ in Eqs. (7) and (8) such that the governing momentum Eq. (3) and thermal Eq. (4) BL equations, written in terms of $\phi(\xi)$ and $\theta(\xi)$, as well as their boundary conditions, do not explicitly involve $\operatorname{Pr}$ and $\mathrm{Ra}$. In this case, the solutions of the resulting BL equations, i.e., the functions $\phi(\xi)$ and $\theta(\xi)$, are also independent of Pr and Ra. In particular, the value of $\theta^{\prime}(0)\left[\phi^{\prime}(\hat{\xi})\right]^{-1 / 2}$ is a pure constant. This together with Eq. (18) immediately imply the following scaling relation for the Nusselt number with the Reynolds and the Prandtl numbers, which thus in general holds for natural boundary-layer dominated large-Prandtl-number thermal convection:

$$
\mathrm{Nu} \sim \operatorname{Re}^{1 / 2} \operatorname{Pr}^{1 / 2}
$$

Note that apart from RBC, the scaling Eq. (20) was found also in other different configurations of natural large-Prandtl-number thermal convective flows, for example, in horizontal convection, where the fluid layer is heated through one region of the bottom and cooled through another region of the bottom [23-25] and also in vertical convection, where the fluid is heated through one vertical surface of the fluid layer and cooled though another vertical surface [22,26].

Previously [5] it was shown that the relation (20) holds also for $\operatorname{Pr} \ll 1$ in laminar Rayleigh-Bénard convection. Since the Pr-dependence as in Eq. (20) holds only for very large or small Pr, it formally breaks down for intermediate values of $\mathrm{Pr}$, which strictly speaking means the absence of the similarity solution. However, also in this case relation (20) provides a fair estimate due to the fact that $\operatorname{Pr} \approx 1$ in this regime and taking this value to some power does not introduce a strong Pr-dependence. Therefore, relation (20) effectively and in good approximation holds for all Prandtl numbers, which is fully supported by our simulations as we will see in the next section. 


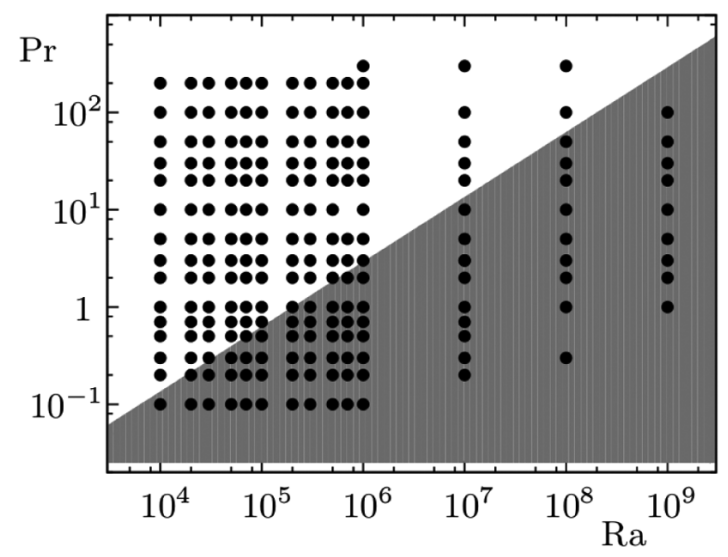

FIG. 1. Sketch of the conducted DNS (symbols) in an (Ra, Pr) plane. White region corresponds to the scaling regimes $\mathrm{I}_{\infty}^{<}$and $\mathrm{III}_{\infty}$. Shadowed region corresponds to transition to other regimes.

\section{SCALINGS OF Re AND Nu WITH Ra AND Pr IN BOUNDARY-LAYER-DOMINATED LARGE-Pr RBC (REGIME I $\left.I_{\infty}^{<}\right)$}

To obtain the second scaling relation, in addition to Eq. (20), we follow Grossmann and Lohse [5] for the BL-dominated thermal convection. The balance of the time- and volume-averaged kinetic dissipation rate $\epsilon_{u}$ to its estimated BL contribution gives $\epsilon_{u} \sim\left(\nu U^{2} / \lambda_{u}^{2}\right)\left(\lambda_{u} / H\right)$, where $\lambda_{u}$ is the thickness of the viscous BL near the bottom plate. This together with Prandtl's relation $\lambda_{u} / H \sim$ $\operatorname{Re}^{-1 / 2}$ for laminar RBC flows [14,15] yields $\epsilon_{u} \sim\left(v^{3} / H^{4}\right) \operatorname{Re}^{5 / 2}$. Thus, from Eq. (1), Eq. (20), and the last relation one obtains the scalings in the laminar low-Pr regime $\mathrm{I}_{\ell}$ of the GL theory:

$$
\begin{aligned}
& \mathrm{Nu} \sim \operatorname{Pr}^{1 / 8} \operatorname{Ra}^{1 / 4}, \\
& \mathrm{Re} \sim \operatorname{Pr}^{-3 / 4} \operatorname{Ra}^{1 / 2} .
\end{aligned}
$$

These scalings have been supported by numerous experimental and numerical RBC studies in the respective regions of the (Pr, Ra) plane, see, e.g., Refs. [11,27-32]; therefore, in the present work we do not focus on the low-Pr regime $\mathrm{I}_{\ell}$.

With decreasing Ra, the viscous BL thickness $\lambda_{u}$ generally increases and slowly saturates to a certain bounding value, which is comparable with $H$ [6]. In this case, the BL contribution to the mean kinetic dissipation rate scales as $\epsilon_{u} \sim\left(\nu U^{2} / H^{2}\right)$, which yields

$$
\epsilon_{u} \sim\left(v^{3} / H^{4}\right) \operatorname{Re}^{2} .
$$

Thus, from Eqs. (1), (20), and (23) the scaling relations in the regime $\mathrm{I}_{\infty}^{<}$of BL-dominated large-Pr RBC follow,

$$
\begin{aligned}
& \mathrm{Nu} \sim \operatorname{Pr}^{0} \mathrm{Ra}^{1 / 3}, \\
& \mathrm{Re} \sim \operatorname{Pr}^{-1} \mathrm{Ra}^{2 / 3} .
\end{aligned}
$$

Note that these obtained scaling relations (24) and (25) are similar to those in the regime $\mathrm{III}_{\infty}[6]$, although in the regime $\mathrm{III}_{\infty}$ the kinetic dissipation turns to be bulk-dominated.

The relations (24) and (25) hold up to very large Pr. The DNS for $\operatorname{Pr}=2548$ and Ra up to $10^{9}$ [33] showed that $\mathrm{Nu} \sim \mathrm{Ra}^{0.3}$ and $\mathrm{Re} \sim \mathrm{Ra}^{0.6}$. Independence of the Nusselt number of the Prandtl number for large Pr was demonstrated also in several other DNS, see, e.g., Refs. [32,34]. The measurements by Xia et al. [35] for Pr from 4 to 1350 and Ra up to $3 \times 10^{10}$ also demonstrated that Nu roughly goes as $\mathrm{Ra}^{0.3}$ and is almost independent of $\mathrm{Pr}$. 

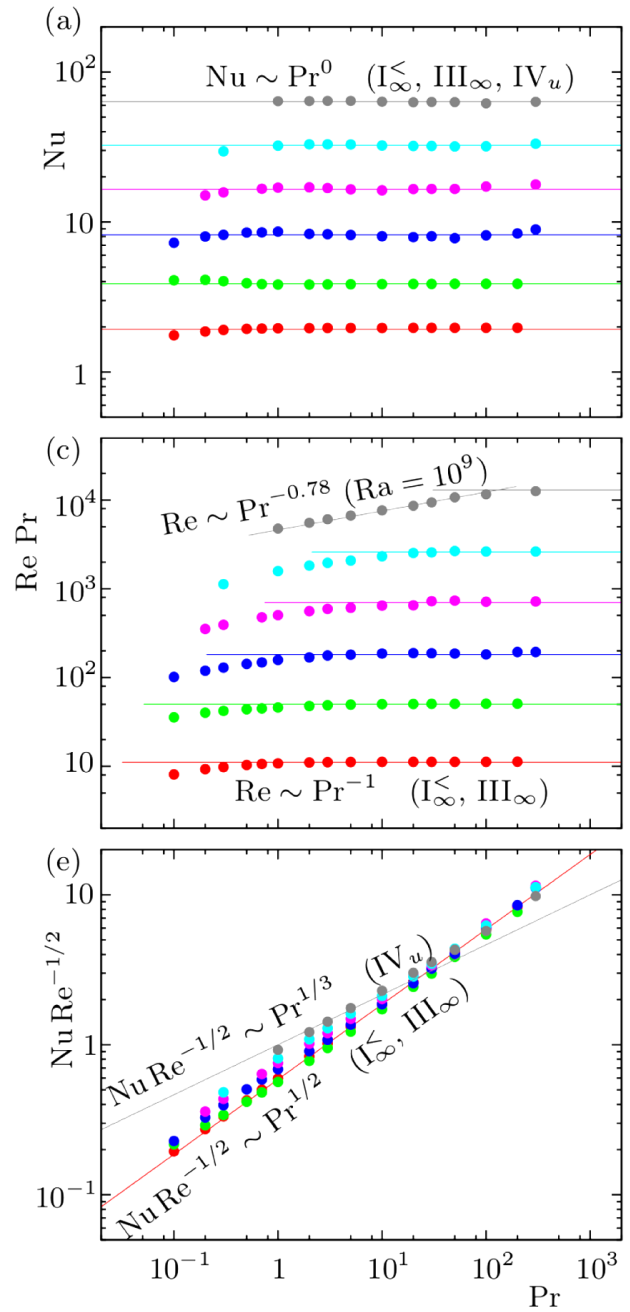
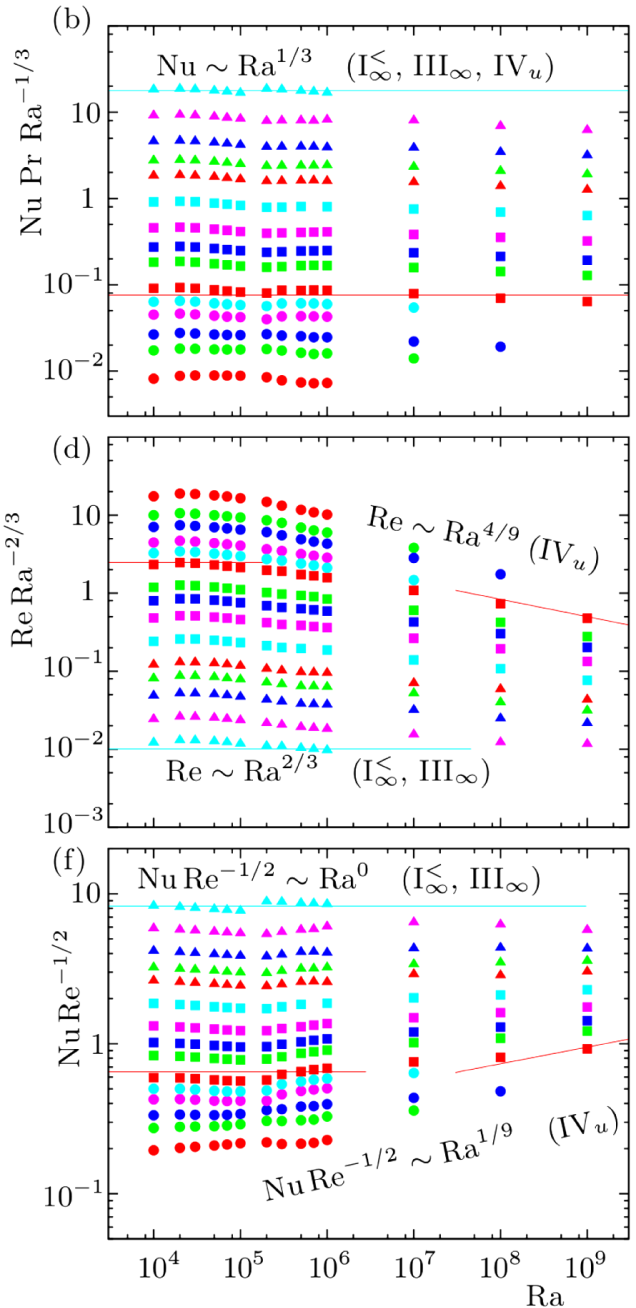

FIG. 2. Nusselt number, Reynolds number and their combinations, as functions of the Prandtl number (a), (c), (e) and of the Rayleigh number (b), (d), (f), as obtained in the DNS of RBC in a cylindrical container of the aspect ratio 1 , for (a), (c), (e) $\mathrm{Ra}=10^{4}$ (red), $\mathrm{Ra}=10^{5}$ (green), $\mathrm{Ra}=10^{6}$ (blue), $\mathrm{Ra}=10^{7}$ (magenta), $\mathrm{Ra}=10^{8}$ (cyan), and $\mathrm{Ra}=10^{9}$ (gray), and for (b), (d), (f) $\mathrm{Pr}=0.1$ (red circles), $\mathrm{Pr}=0.2$ (green circles), $\operatorname{Pr}=0.3$ (blue circles), $\operatorname{Pr}=0.5$ (magenta circles), $\operatorname{Pr}=0.7$ (cyan circles), $\operatorname{Pr}=1$ (red squares), $\operatorname{Pr}=2$ (green squares), $\operatorname{Pr}=3$ (blue squares), $\operatorname{Pr}=5$ (magenta squares), $\operatorname{Pr}=10$ (cyan squares), $\operatorname{Pr}=20$ (red triangles), $\operatorname{Pr}=30$ (green triangles), $\operatorname{Pr}=50$ (blue triangles), $\operatorname{Pr}=100$ (magenta triangles), $\operatorname{Pr}=200$ (cyan triangles).

The results of our present DNS, which were conducted using the finite-volume code goldfish (see, e.g., Refs. [17,36] and Fig. 1) for RBC for Ra from $10^{4}$ to $10^{9}$ and Pr from 0.1 to 200, are summarized in Fig. 2. In the left column [Figs. 2(a), 2(c), and 2 (e)] the Prandtl number dependencies and in the right column [Figs. 2(b), 2(d) and 2(f)] the Rayleigh number dependencies are presented for the Nusselt number [Figs. 2(a) and 2(b)], Reynolds number [Figs. 2(c) and 2(d)], and their combination $\mathrm{Nu} \mathrm{Re} e^{-1 / 2}$, due to the relation (20).

One can see that through several decades of Pr and Ra, the Nusselt number remains to be independent of Pr [Fig. 2(a)] and scales with the Rayleigh number as $\sim \mathrm{Ra}^{1 / 3}$ [Fig. 2(b)], in full agreement with Eq. (24). Note that also for the larger Ra this scaling should hold, as on this end the 
regime $\mathrm{III}_{\infty}$ enters the regime $\mathrm{IV}_{u}[5,6]$ with its scaling relations:

$$
\begin{gathered}
\mathrm{Nu} \sim \operatorname{Pr}^{0} \operatorname{Ra}^{1 / 3}, \\
\operatorname{Re} \sim \operatorname{Pr}^{-2 / 3} \operatorname{Ra}^{4 / 9} .
\end{gathered}
$$

For the smallest $\operatorname{Pr}[\operatorname{Pr}<0.5$ in Fig. 2(a)], the Nusselt number slightly grows with $\operatorname{Pr}$, as the flow undergoes a transition from the regime $\mathrm{I}_{\infty}^{<}$to the regime $\mathrm{I}_{\ell}$, with its own scaling Eq. (21).

From Fig. 2(c), we can conclude the following: For sufficiently large $\operatorname{Pr}(\operatorname{Pr} \geqslant 0.5)$ and moderate $\mathrm{Ra}\left(\mathrm{Ra}<10^{9}\right)$, the Reynolds number scales as $\operatorname{Re} \sim \operatorname{Pr}^{-1}$ through several decades of $\operatorname{Pr}$, as it should be in the regimes $\mathrm{I}_{\infty}^{<}$and $\mathrm{III}_{\infty}$; see Eq. (25). Again, the region of very small $\operatorname{Pr}$, $\operatorname{Pr}<0.5$, belongs to the scaling regime $\mathbf{I}_{\ell}$ Eq. (22) and, therefore, the values of (Re Pr) increase with increasing Pr. The large-Ra region is already around the transition to the regime $\mathrm{IV}_{u}$, where the scaling Eq. (27) should take over. For $\mathrm{Ra}=10^{9}$, the Reynolds number behaves already as $\operatorname{Re} \sim \operatorname{Pr}^{-0.78}$, as our simulations show. Figure 2(d) also supports the scaling Eq. (25), but this time with respect to the Ra-scaling in large-Pr BL-dominated RBC. Indeed, $\mathrm{Re}$ goes as $\sim \mathrm{Ra}^{2 / 3}$ there, with a tendency to $\sim \mathrm{Ra}^{4 / 9}$ Eq. (27), as it should be in the scaling regime $\mathrm{IV}_{u}$ [this slope is shown in Fig. 2(d) with a red inclined line].

Finally, in Fig. 2(f) one can see that $\mathrm{Nu} \mathrm{Re}^{-1 / 2}$ in the regimes $\mathrm{I}_{\infty}^{<}$and $\mathrm{III}_{\infty}$ is independent of Ra. For larger Ra, this quantity starts to increase and tends to $\mathrm{Nu} \mathrm{Re}^{-1 / 2} \sim \mathrm{Ra}^{1 / 9}$, as it should be in the scaling regime $\mathrm{IV}_{u}$ [this slope is shown in Fig. 2(f) with a red inclined line]. The Prandtl-number dependencies of $\mathrm{NuRe}^{-1 / 2}$ in Fig. 2(e) also support the scaling relations (24) and (25) for the regimes $\mathrm{I}_{\infty}^{<}$and $\mathrm{III}_{\infty}$ and the scaling relations (26) and (27) for the regime $\mathrm{IV}_{u}$, i.e., it varies from $\mathrm{Nu} \operatorname{Re}^{-1 / 2} \sim \operatorname{Pr}^{1 / 2}$ for smaller $\operatorname{Ra}$ to $\mathrm{Nu} \operatorname{Re}^{-1 / 2} \sim \operatorname{Pr}^{1 / 3}$ for larger Ra.

\section{CONCLUSIONS}

In the present work we derived that the relation $\mathrm{Nu} \sim \operatorname{Re}^{1 / 2} \operatorname{Pr}^{1 / 2}$ holds in laminar natural thermal convection, where no wind is imposed above the isothermal plate, for large Prandtl numbers $\operatorname{Pr} \gg 1$. Our derivation is based on a generalization of the Prandtl approach [12] and on a search of a similarity solution of the laminar thermal BL equation when $\operatorname{Pr} \rightarrow \infty$. The scaling relation (20), $\mathrm{Nu} \sim \operatorname{Re}^{1 / 2} \operatorname{Pr}^{1 / 2}$, which holds for all Prandtl numbers in laminar Rayleigh-Bénard convection, holds more generally also in the non-laminar regimes. It strictly holds for very large or very small $\operatorname{Pr}$, but formally breaks down for intermediate Pr. However, as in this case $\operatorname{Pr} \sim 1$, the relation $\mathrm{Nu} \sim \operatorname{Re}^{1 / 2} \operatorname{Pr}^{1 / 2}$ still provides a good approximation of the relationship between $\mathrm{Re}, \mathrm{Pr}$, and $\mathrm{Nu}$ also in this regime, which is fully supported by our simulations in a wide parameter range.

Because of the relation (20), the limiting large-Pr laminar regime in $\mathrm{RBC}$ is regime $\mathrm{I}_{\infty}^{<}$with the scaling relations (24) and (25), which were originally derived in Ref. [6] as one of the possible scaling regimes in large-Pr thermal convection.

Based on our DNS data for Ra from $10^{4}$ to $10^{9}$ and Pr from 0.1 to 200 (totally 200 cases), we showed that the scaling regime $\mathrm{I}_{\infty}^{<}$undergoes a smooth transition into regime $\mathrm{III}_{\infty}$, so that one does not necessarily have to distinguish them. For sufficiently large $\mathrm{Ra}$, the scaling relations in regime $\mathrm{III}_{\infty}$ undergo a transition to those of regime $\mathrm{IV}_{u}$. All these scaling relations and transitions have been supported by our DNS over a large range of Ra and Pr.

\section{ACKNOWLEDGMENTS}

O.S. acknowledges financial support the German Research Foundation (DFG) under Grants No. Sh405/3-2 and No. Sh405/4-2 (Heisenberg fellowship) and the Leibniz Supercomputing Centre (LRZ) for providing computing time. We also acknowledge support by the Netherlands Organisation for Scientific Research (NWO) and the Max Planck Center for Complex Fluid Dynamics. 
[1] G. Ahlers, S. Grossmann, and D. Lohse, Heat transfer and large scale dynamics in turbulent RayleighBénard convection, Rev. Mod. Phys. 81, 503 (2009).

[2] E. Bodenschatz, W. Pesch, and G. Ahlers, Recent developments in Rayleigh-Bénard convection, Annu. Rev. Fluid Mech. 32, 709 (2000).

[3] D. Lohse and K.-Q. Xia, Small-scale properties of turbulent Rayleigh-Bénard convection, Annu. Rev. Fluid Mech. 42, 335 (2010).

[4] F. Chillà and J. Schumacher, New perspectives in turbulent Rayleigh-Bénard convection, Eur. Phys. J. E 35, 58 (2012).

[5] S. Grossmann and D. Lohse, Scaling in thermal convection: A unifying theory, J. Fluid Mech. 407, 27 (2000).

[6] S. Grossmann and D. Lohse, Thermal Convection for Large Prandtl Numbers, Phys. Rev. Lett. 86, 3316 (2001).

[7] S. Grossmann and D. Lohse, Prandtl and Rayleigh number dependence of the Reynolds number in turbulent thermal convection, Phys. Rev. E 66, 016305 (2002).

[8] S. Grossmann and D. Lohse, On geometry effects in Rayleigh-Bénard convection, J. Fluid Mech. 486, 105 (2003).

[9] S. Grossmann and D. Lohse, Fluctuations in turbulent Rayleigh-Bénard convection: The role of plumes, Phys. Fluids 16, 4462 (2004).

[10] S. Grossmann and D. Lohse, Multiple scaling in the ultimate regime of thermal convection, Phys. Fluids 23, 045108 (2011).

[11] R. J. A. M. Stevens, E. P. van der Poel, S. Grossmann, and D. Lohse, The unifying theory of scaling in thermal convection: The updated prefactors, J. Fluid Mech. 730, 295 (2013).

[12] L. Prandtl, Über Flüssigkeitsbewegung bei sehr kleiner Reibung, in Verhandlungen des III. Int. Math. Kongr., Heidelberg, 1904 (Teubner, Munich, 1905), pp. 484.

[13] H. Blasius, Grenzschichten in Flüssigkeiten mit kleiner Reibung, Z. Math. Phys. 56, 1 (1908).

[14] L. D. Landau and E. M. Lifshitz, Fluid Mechanics, 2nd ed., Course of Theoretical Physics, Vol. 6 (Butterworth Heinemann, Oxford, UK, 1987).

[15] H. Schlichting and K. Gersten, Boundary-Layer Theory, 8th ed. (Springer, Berlin, 2000).

[16] Q. Zhou and K.-Q. Xia, Measured Instantaneous Viscous Boundary Layer in Turbulent Rayleigh-Bénard Convection, Phys. Rev. Lett. 104, 104301 (2010).

[17] O. Shishkina, S. Horn, S. Wagner, and E. S. C. Ching, Thermal Boundary Layer Equation for Turbulent Rayleigh-Bénard convection, Phys. Rev. Lett. 114, 114302 (2015).

[18] E. S. C. Ching, O.-Y. Dung, and O. Shishkina, Fluctuating thermal boundary layers and heat transfer in turbulent Rayleigh-Bénard convection, J. Stat. Phys. 167, 626 (2017).

[19] E. S. C. Ching, Heat flux and shear rate in turbulent convection, Phys. Rev. E 55, 1189 (1997).

[20] O. Shishkina, S. Horn, and S. Wagner, Falkner-Skan boundary layer approximation in Rayleigh-Bénard convection, J. Fluid Mech. 730, 442 (2013).

[21] O. Shishkina, S. Wagner, and S. Horn, Influence of the angle between the wind and the isothermal surfaces on the boundary layer structures in turbulent thermal convection, Phys. Rev. E 89, 033014 (2014).

[22] O. Shishkina, Momentum and heat transport scalings in laminar vertical convection, Phys. Rev. E 93, 051102(R) (2016).

[23] O. Shishkina and S. Wagner, Prandtl-Number Dependence of Heat Transport in Laminar Horizontal Convection, Phys. Rev. Lett. 116, 024302 (2016).

[24] O. Shishkina, S. Grossmann, and D. Lohse, Heat and momentum transport scalings in horizontal convection, Geophys. Res. Lett. 43, 1219 (2016).

[25] G. O. Hughes and R. W. Griffiths, Horizontal convection, Annu. Rev. Fluid Mech. 40, 185 (2008).

[26] C. S. Ng, A. Ooi, D. Lohse, and D. Chung, Vertical natural convection: Application of the unifying theory of thermal convection, J. Fluid Mech. 764, 0349 (2015).

[27] R. M. Kerr, Rayleigh number scaling in numerical convection, J. Fluid Mech. 310, 139 (1996).

[28] S. Cioni, S. Ciliberto, and J. Sommeria, Strongly turbulent Rayleigh-Bénard convection in mercury: Comparison with results at moderate Prandtl number, J. Fluid Mech. 335, 111 (1997). 
[29] R. Verzicco and R. Camussi, Prandtl number effects in convective turbulence, J. Fluid Mech. 383, 55 (1999).

[30] R. Verzicco and R. Camussi, Numerical experiments on strongly turbulent thermal convection in a slender cylindrical cell, J. Fluid Mech. 477, 19 (2003).

[31] E. P. van der Poel, R. J. A. M. Stevens, K. Sugiyama, and D. Lohse, Flow states in two-dimensional Rayleigh-Bénard convection as a function of aspect ratio and Rayleigh number, Phys. Fluids 24, 085104 (2012).

[32] K. Petschel, S. Stellmach, M. Wilczek, J. Lülff, and U. Hansen, Dissipation Layers in Rayleigh-Bénard Convection: A unifying View, Phys. Rev. Lett. 110, 114502 (2013).

[33] S. Horn, O. Shishkina, and C. Wagner, On non-Oberbeck-Boussinesq effects in three-dimensional Rayleigh-Bénard convection in glycerol, J. Fluid Mech. 724, 175 (2013).

[34] E. P. van der Poel, R. J. A. M. Stevens, and D. Lohse, Comparison between two- and three-dimensional Rayleigh-Bénard convection, J. Fluid Mech. 736, 177 (2013).

[35] K.-Q. Xia, S. Lam, and S. Q. Zhou, Heat-Flux Measurement in High-Prandtl-Number Turbulent RayleighBénard Convection, Phys. Rev. Lett. 88, 064501 (2002).

[36] O. Shishkina and S. Horn, Thermal convection in inclined cylindrical containers, J. Fluid Mech. 790, R3 (2016). 